\title{
Mapping QTLs for Fertility Restoration of Different Cytoplasmic Male Sterility Types in Rice Using Two Oryza sativa $\times$ O. rufipogon Backcross Inbred Line Populations
}

\author{
Biao-lin Hu, ${ }^{1,2}$ Jian-kun Xie, ${ }^{1}$ Yong Wan, ${ }^{2}$ Jin-wei Zhang, ${ }^{1}$ Fan-tao Zhang, ${ }^{1}$ and Xia Li ${ }^{2}$ \\ ${ }^{1}$ College of Life Science, Jiangxi Normal University, Nanchang, Jiangxi 330022, China \\ ${ }^{2}$ Rice Research Institute, Jiangxi Academy of Agricultural Sciences and Nanchang National Sub-Center for Rice Improvement, \\ Nanchang, Jiangxi 330200, China \\ Correspondence should be addressed to Jian-kun Xie; xiejiankun11@163.com
}

Received 16 July 2016; Accepted 5 October 2016

Academic Editor: Seonghee Lee

Copyright (C) 2016 Biao-lin Hu et al. This is an open access article distributed under the Creative Commons Attribution License, which permits unrestricted use, distribution, and reproduction in any medium, provided the original work is properly cited.

\begin{abstract}
Hybrid rice breeding using cytoplasmic male sterility/fertility restoration (CMS/Rf) systems plays an important role in ensuring global food security. Two backcross inbred line (BIL) populations derived from either Xieqingzao B (XB)//XB/Dongxiang wild rice (DWR) (XXD) or XB//DWR/XB (XDX) were used to detect quantitative trait loci (QTLs) for fertility restoration of Dwarf wild abortive- (DA-), Indonesia Paddy- (ID-), and DWR-type CMS in rice. Lines with ID- and DA-type CMS were testcrossed with both the XXD- and XDX-BILs, while the line with DWR-type CMS was testcrossed with the XDX-BILs only. A total of 16 QTLs for fertility restoration of CMS systems were identified, including three for DWR-type CMS, six for DA-type CMS, and seven for ID-type CMS. All of the additive alleles in the QTLs were derived from Oryza rufipogon. Eleven QTLs were clustered in five chromosomal regions, indicating that common $R f$ loci restored different CMS systems, and the favorable O. rufipogon alleles could be used to develop restorer lines for various CMS types by marker-assisted selection.
\end{abstract}

\section{Introduction}

Rice (Oryza sativa L.) is one of the most important staple crops worldwide, providing $21 \%$ of the food calorie supply for the world's population and up to $76 \%$ of that for Southeast Asia [1]. Thus, the improvement of global food security relies heavily on the ability to sustainably increase rice yields [2,3]. Such increases are possible through the use of novel highyielding varieties of rice, which will depend in turn on the development of heterotic rice hybrids [4].

Hybrids often exhibit superior growth traits than either parental line, due to what is referred to as heterosis or hybrid vigor [5]. Cytoplasmic male sterility/fertility restoration $(\mathrm{CMS} / R f)$ systems provide a powerful approach in the production of hybrid seed [6]. CMS/Rf systems are widespread in flowering plant species. CMS caused by an incompatibility between mitochondrial and nuclear genomes results in unviable pollen, preventing seed development in a self-pollinating plant like rice. CMS can however be rescued by nuclear $R f$ gene(s) [7-9]. Therefore, in rice breeding programs, the combination of CMS in the female parent and $R f$ gene(s) in the male parent is indispensable for the commercial development of three-line hybrid rice.

Since the initial discovery of CMS in a rice line that possessed the Chinsurah Boro II cytoplasm, over 60 types of CMS associated with different cytoplasmic resources have been reported [10]. In rice, thirteen types of CMS have been commercially used for hybrid rice production, namely, those from Boro II (BT), Dwarf wild abortive (DA), Dissi (D), Dianl (Dianl), Gambiaka (G), Honglian (HL), Indonesia Paddy (ID), K52 (K), Luihui (LX), Maxie (Maxie), NX (NX), wild abortive (WA), and Yegong (Y) [11]. Although the use of WA-type CMS is still dominant, there has been a gradual increase in the proportion of ID- and DA-type CMS used for hybrid rice production $[11,12]$. However, relatively little is known regarding the origins, evolutionary relationships, and distribution of $R f$ genes for ID- and DA-type CMS. 
In general, CMS can be restored by one or two nuclear $R f$ genes. For example, in WA-type CMS, pollen abortion caused by the WA352 gene is rescued by nuclear genes $R f 3$ and $R f 4$, which are located on chromosomes 1 and 10 , respectively [8]. A large number of reports have focused on the mapping of $R f$ genes or loci that rescue various CMS types, such as those from $\mathrm{BT}$, Chinese wild rice $(\mathrm{CW}), \mathrm{D}$, DA, Dian1, G, HL, Lead Rice (LD), ID, and WA [13-19]. In these studies, $R f$ genes were detected by bulk segregant analysis of an $\mathrm{F}_{2}$ or backcross population. To date, more than $17 R f$ genes or loci that rescue different CMS types have been identified, distributed across all rice chromosomes apart from chromosome 9 (Gramene, http://www.gramene.org). Of these, seven $R f$ genes have been identified and functionally characterized: $R f 1$ ( $R f 1 a$ and $R f 1 b$ ) for BT-type CMS [20, 21], $R f 2$ for LD-type CMS [22], $R f 4$ for WA-type CMS [23, 24], Rf5 and Rf6 for HL-CMS [25, 26], and Rf17 for CW-CMS [27]. Molecular cloning and characterization of these $R f$ genes not only further described the cytoplasmic-nuclear interactions, but also provided powerful molecular tools to assist in hybrid breeding by accelerating the development of novel restorer lines. However, Rf7 to Rf16 remain to be characterized, suggesting that further study is needed on the genetic effect of $R f$ genes [28]. Furthermore, although five of the seven characterized $R f$ genes (all except $R f 2$ and $R f 17$ ) are used in commercial hybrid rice production in China, they all originate from indica rice. The current limit in yield from three-line hybrid rice in China might be explained by the small number of $R f$ genes used to develop restorer lines in hybrid rice breeding [12]. Thus, exploration of new $R f$ genes will greatly enrich the resources available to improve rice yields using heterosis. Furthermore, the exact number of different $R f$ genes that exist for a particular CMS type is still unclear. Therefore, the discovery of new $R f$ loci or alleles in wild rice not only will add to the current genetic resources used in CMS/Rf systems for hybrid rice, but also will lead to a better understanding of the origin and evolution of $R f$ genes in rice.

Dongxiang wild rice (Oryza rufipogon Griff., hereafter referred to as DWR) is a common wild rice variety distributed in northern regions worldwide. In addition to its remarkable traits for cold and drought tolerance $[29,30]$, DWR carries $R f$ genes for CMS [31]. Therefore, there is potential to identify these valuable $R f$ genes and develop them for use in hybrid rice breeding. In the present study, an interspecific cross between a rice cultivar and an accession of DWR was conducted to detect QTLs for fertility restoration of various CMS types. The objective of this work was to identify potential alleles from $O$. rufipogon to add to the $R f$ genes currently used in CMS/Rf systems for hybrid rice.

\section{Materials and Methods}

2.1. Plant Materials. Two mapping populations consisting of either 202 or 237 backcross inbred lines (BILs) were derived from a backcross using rice cultivar Xieqingzao B (Oryza sativa L., hereafter referred to as $\mathrm{XB}$ ) as the recurrent parent and an accession of DWR as the donor parent. The development process of $202 \mathrm{BC}_{1} \mathrm{~F}_{5}$ BILs derived from cross
$\mathrm{XB} / / \mathrm{XB} / \mathrm{DWR}$ (hereafter referred to as $\mathrm{XXD}$ ) was described by Chen et al. [32]. The $237 \mathrm{BC}_{1} \mathrm{~F}_{10}$ BILs were established by first crossing an accession of DWR as the female parent with cultivated rice $\mathrm{XB}$ to generate an $\mathrm{F}_{1}$ hybrid in 1998 by Rice Research Institute, Jiangxi Academy of Agricultural Sciences (RRI, JAAS), Nanchang, China. Following this, the $\mathrm{F}_{1}$ plants were backcrossed to $\mathrm{XB}$ to generate a $\mathrm{BC}_{1} \mathrm{~F}_{1}$ cross of $\mathrm{XB} / / \mathrm{DWR} / \mathrm{XB}$ (hereafter referred to as XDX), from which a BIL population of $237 \mathrm{BC}_{1} \mathrm{~F}_{10}$ lines was obtained by single seed descent in 2008.

A controlled cross between three alloplasmic CMS lines, including Xieqingzao A (XA), Zhong 9A (ZA), and DongB11 $\mathrm{A}(\mathrm{DbA})$, and each line of the $\mathrm{BC}_{1} \mathrm{~F}_{5} \mathrm{XXD}-\mathrm{BIL}$ and $\mathrm{BC}_{1} \mathrm{~F}_{10}$ XDX-BIL population was made, forming five sets of the testcross populations. As a result, two and three sets of the $\mathrm{F}_{1}$ testcross populations (XA/XXD-BIL and ZA/XXD-BIL, and DbA/XDX-BIL, XA/XDX-BIL, and ZA/XDX-BIL) were obtained in 2010 and 2014, respectively. Xieqingzao A is a typical DA-type CMS line of superhybrid rice Xieyou9308. Zhong 9A is a typical ID-type CMS line of superhybrid rice Zhongyou228. DongB11 A is a CMS line with DWR cytoplasm of superhybrid rice Dongyou 962, which was bred by Xiao et al. [33].

2.2. Field Experiments and Phenotyping. In the rice-growing seasons from May to October in 2010 and 2014, the phenotyping experiments were conducted in experimental fields at the Rice Research Institute, Jiangxi Academy of Agricultural Sciences in Nanchang, Jiangxi (latitude: $28^{\circ} 33^{\prime} \mathrm{N}$, longitude: $115^{\circ} 56^{\prime} \mathrm{E}$ ). In 2010 experiment, two sets of testcross populations consisting of $202 \mathrm{XA} / \mathrm{XXD}$-BIL and ZA/XXD-BIL lines and the $\mathrm{F}_{1}$ progeny from crosses between $\mathrm{XB}$ and two CMS lines were grown. In 2014 experiment, three sets of testcross populations consisting of $237 \mathrm{DbA} / \mathrm{XDX}-\mathrm{BIL}, \mathrm{XA} / \mathrm{XDX}-\mathrm{BIL}$, and $\mathrm{ZA} / \mathrm{XDX}-\mathrm{BIL}$ lines and the $\mathrm{F}_{1}$ progeny from crosses between parent $\mathrm{XB}$ and three CMS lines were grown. In field trials, each line was transplanted to plots with a spacing of $16.7 \mathrm{~cm}$ between plants within a row and $26.7 \mathrm{~cm}$ between rows. Each plot consisted of three rows with 12 plants per row. The field trial was managed with the normal agricultural practice.

At maturity, five plants from the middle of each line plot were harvested, unless high sterility was observed, in which case only one plant was harvested. For phenotyping of the fertility restoration, spikelet fertility (SF) was estimated as the ratio (percentage) of filled grains to the total number of grains per panicle. The average SF value of the randomly sampled plants from each line was used as a measure of fertility restoration for data analysis.

2.3. DNA Marker Analysis and Genetic Map Construction. The $237 \mathrm{BC}_{1} \mathrm{~F}_{10}$ BILs and the recurrent parent $\mathrm{XB}$ were grown in 2009 in Nanchang. The fresh leaves from a single plant per line were collected for total genomic DNA extraction according to Zheng et al. [36]. A total of 300 SSR and 50 InDel markers were used to survey parental polymorphism. All of SSR markers were obtained from GRAMENE (http://www.gramene.org). The InDel markers were designed according to the DNA length polymorphism of 
TABLE 1: Performance of spikelet fertility in the testcross populations and $\mathrm{F}_{1}$ plants from Xieqingzao B (XB) crossed with CMS lines.

\begin{tabular}{|c|c|c|c|c|c|c|c|c|}
\hline \multirow{2}{*}{ Year } & \multirow{2}{*}{ Combination } & \multirow{2}{*}{$\mathrm{F}_{1}$ fertility $^{\mathrm{a}}(\%)$} & \multicolumn{6}{|c|}{ Testcross population } \\
\hline & & & Mean (\%) & $\mathrm{SD}$ & $\mathrm{CV}$ & Range (\%) & Skewness & Kurtosis \\
\hline \multirow{3}{*}{2010} & ZA/XXD-BIL & 5.1 & 17.5 & 13.7 & 0.78 & $1.1-90.4$ & 2.0 & 6.0 \\
\hline & XA/XXD-BIL & 0.6 & 15.0 & 12.9 & 0.86 & $1.4-82.6$ & 2.6 & 9.2 \\
\hline & ZA/XDX-BIL & 7.5 & 35.7 & 22.26 & 0.62 & $1.8-88.9$ & 0.55 & -0.81 \\
\hline \multirow[t]{2}{*}{2014} & XA/XDX-BIL & 1.1 & 38.8 & 23.46 & 0.60 & $3.0-93.2$ & 0.46 & -0.87 \\
\hline & DbA/XDX-BIL & 20.0 & 34.9 & 22.09 & 0.63 & $2.6-89.2$ & 0.44 & -0.92 \\
\hline
\end{tabular}

${ }^{a} \mathrm{~F}_{1}$ fertility means the average $\mathrm{F}_{1}$ fertility derived from parent Xieqingzao B and CMS lines (ZA, XA, and DbA).

Nipponbare and 93-11 (https://www.ncbi.nlm.nih.gov/) using Premier 5.0 software. As the original DWR accession is no longer available, three DNA bulks, each consisting of 10 randomly selected BILs, and the DNA from the parent XB were used.

Polymerase chain reaction (PCR) was performed in a $10 \mu \mathrm{L}$ reaction mixture containing $5.0 \mu \mathrm{L}$ of 2 Taq Master Mix (CWBIO, Beijing, China), 3 MlRnase-free $\mathrm{H}_{2} \mathrm{O}$ (CWBIO, Beijing, China), $1 \mu \mathrm{L}$ template DNA, and $1 \mu \mathrm{L}$ of $3.3 \mathrm{ng} \mu \mathrm{L}^{-1}$ SSR primers. The cycling profile for the PCR consisted of initial denaturation at $94^{\circ} \mathrm{C}$ for $2 \mathrm{~min}, 30$ cycles at $94^{\circ} \mathrm{C}$ for $30 \mathrm{~s}, 55^{\circ} \mathrm{C}$ for $30 \mathrm{~s}$, and $72^{\circ} \mathrm{C}$ for $30 \mathrm{~s}$, followed by a final extension at $72^{\circ} \mathrm{C}$ for $2 \mathrm{~min}$. The PCR products were separated on $2.5 \%$ agarose gel stained with Gelred (Biotium, Hayward, CA, USA) and recorded using a MiniBIS Pro (DNR BioImaging Systems Ltd., Jerusalem, Israel) or 6\% nondenaturing polyacrylamide gel and silver staining, depending on the sizes of the DNA fragments and clarity of the bands [36].

2.4. Linkage Map Construction and Data Analysis. Linkage map for the XXD population was firstly constructed by Chen et al. [32] and updated by Huang et al. [37]. For the XDX population, each BIL was genotyped with 145 polymorphic markers, including 12 InDels and 133 SSRs. The linkage map was constructed using Mapmaker/Exp 3.0 software [38]. Distances between markers were estimated using the Kosambi function and presented in centiMorgan (cM).

Descriptive statistics of phenotypic data, including minimum and maximum trait values, mean trait value, standard deviation, coefficient of variation, skewness, and kurtosis, were calculated using the command DSum of the software Windows QTL Cartographer 2.5 [39].

QTLs for fertility restoration were detected using Windows QTL Cartographer 2.5 [39]. Composite interval mapping was performed using a walking speed of $1 \mathrm{cM}$ and a window size of $10 \mathrm{cM}$ with backward and forward regression. A logarithm of the odds (LOD) threshold $>2.0$ was used to claim a putative QTL. The QTL was designated following the nomenclature commended by McCouch and CGSNL [40].

\section{Results}

3.1. Phenotypic Performance. Descriptive statistics of spikelet fertility are summarized in Table 1 . Wide variations of spikelet fertility with continuous distribution were observed in each of the five $\mathrm{F}_{1}$ testcross populations.
For the $F_{1}$ testcross population sets grown in 2010, average spikelet fertility of the testcross population ZA/XXD was higher than that of the XA/XXD population. Positive correlation with moderate coefficients of $0.606(P<0.01)$ was observed between the test populations. For the $\mathrm{F}_{1}$ testcross population sets grown in 2014, average spikelet fertility in the testcross population XA/XDX was highest, followed by lower spikelet fertility in the ZA/XDX and lower still in the DbA/XDX population. Highly significant $(P<0.01)$ and positive correlations were shown between $\mathrm{DbA} / \mathrm{XDX}$-BIL and ZA/XDX-BIL populations, DbA/XDXBIL and XA/XDX-BIL populations, and ZA/XDX-BIL and XA/XDX-BIL populations, with coefficients of $0.611,0.596$, and 0.554 , respectively.

3.2. Construction of Linkage Map. Of the 300 SSR and 50 InDel markers examined, 135 SSR and 18 InDel markers were shown to be polymorphic, for a ratio of $43.71 \%$, and used for genotyping of the 237 XDX-BILs. A linkage map of the XDX population was constructed using 133 SSRs and 12 InDels and spanned $1620.9 \mathrm{Cm}$ of the 12 rice chromosomes with an average distance of $11.2 \mathrm{cM}$ between adjacent markers.

3.3. QTLs Determination. A total of 16 QTLs for fertility restoration of three types of CMS were detected, including three for DWR-type CMS, six for the DA-type CMS, and seven for the ID-type CMS (Table 2 and Figure 1). They were distributed on rice chromosomes 1, 3, 5, 7, 9, and 10. qRf7 and $q R$ f10.1 for ID-type CMS were found in the interval RM5752RM6574 on chromosome 7 and in the vicinity of RM5620 on chromosome 10 in the XDX- and XXD-BIL, respectively. The proportion of phenotypic variance explained $\left(R^{2}\right)$ by a single QTL ranged as $11.7 \%-54.9 \%$ for DWR-type CMS, $8.2 \%-49.3 \%$ for DA-type CMS, and $4.9 \%-44.3 \%$ for ID-type CMS, respectively.

In the $\mathrm{F}_{1}$ testcross population of DbA/XDX-BIL, three QTLs for fertility restoration were detected. Individually, these QTLs explained $11.7 \%-54.9 \%$ of the phenotypic variance and had additive effects ranging from $8.8 \%$ to $17.2 \%$. The trait-enhancing alleles were all from DWR. Of these, the QTL $q R f 10.2$ had the largest LOD score of 22.78 and contributed $54.9 \%$ to the phenotypic variance, with the DWR allele increasing spikelet fertility by $17.2 \%$.

For DA-type CMS, six QTLs were detected for fertility restoration, including two detected in the XA/XDX-BILs population and four in the XA/XXD-BIL population. For 
TABLE 2: QTLs conferring fertility restoration for DA-, DWR-, and ID-type CMS detected in the two sets of BIL populations.

\begin{tabular}{|c|c|c|c|c|c|c|}
\hline Population & QTL & Interval & LOD & $A$ & $R^{2}$ & Previous reports \\
\hline \multirow{4}{*}{ DbA/XDX-BIL } & $q R f 1.1$ & RM428-RM10176 & 7.63 & 11.6 & 13.2 & \\
\hline & $q R f 5.1$ & RM188-RM3870 & 4.84 & 8.8 & 11.7 & \\
\hline & $q R f 10.2$ & RM171-RM590 & 22.78 & 17.2 & 54.9 & \\
\hline & $q R f 1.2$ & RM10176-RM243 & 6.65 & 17.6 & 38.9 & [19] \\
\hline XA/XDX-BIL & $q R f 10.2$ & RM171-RM590 & 11.59 & 17.8 & 49.3 & {$[19,34]$} \\
\hline \multirow{5}{*}{ XA/XXD-BIL } & $q R f 1.3$ & RM9-RM3475 & 4.86 & 6.1 & 15.1 & \\
\hline & $q R f 1.4$ & RM315-RG236 & 9.00 & 19.7 & 30.2 & \\
\hline & $q R f 5.2$ & RM87-RM334 & 4.28 & 5.3 & 8.2 & [19] \\
\hline & $q R f 9$ & RM1896-RM201 & 2.28 & 6.8 & 16.0 & \\
\hline & $q R f 3$ & RM282-RM16 & 4.17 & 10.9 & 14.9 & \\
\hline \multirow{4}{*}{ ZA/XDX-BIL } & $q R f 5.1$ & RM188-RM3870 & 5.59 & 11.1 & 17.4 & \\
\hline & $q R f 7$ & RM481-Indel 7.1 & 3.28 & 11.3 & 9.3 & \\
\hline & $q R f 10.1$ & RM1375-RM5620 & 3.70 & 9.1 & 13.2 & \\
\hline & $q R f 10.2$ & RM171-RM590 & 13.33 & 17.2 & 44.3 & {$[14]$} \\
\hline \multirow{4}{*}{ ZA/XXD-BIL } & $q R f 1.4$ & RM315-RG236 & 6.58 & 14.9 & 27.5 & {$[35]$} \\
\hline & $q R f 5.2$ & RM334-RG119 & 2.34 & 4.0 & 4.9 & \\
\hline & $q R f 7$ & RM5752-RM6574 & 3.13 & 6.9 & 10.0 & \\
\hline & $q R f 10.1$ & RM5620-RM1125 & 6.30 & 6.2 & 14.0 & {$[14]$} \\
\hline
\end{tabular}

$A$ indicates an additive effect of replacing a Xieqingzao B allele by a Dongxiang wild rice allele; $R^{2}$ indicates the proportion of phenotypic variance explained by the QTL effect.

qRf5.1, which had the lowest $R^{2}$ of $8.2 \%$, the DWR alleles increased spikelet fertility by $5.3 \%$. For the remaining five QTLs, which had $R^{2}$ ranging from $15.1 \%$ to $49.3 \%$, the DWR alleles improved spikelet fertility by $6.1 \%-17.8 \%$.

For ID-type CMS, seven QTLs were detected for fertility restoration, including two detected in both the ZA/XDX-BIL and ZA/XXD-BIL, three detected in the ZA/XDX-BIL only, and two detected in the ZA/XXD-BIL only. The common QTL $q R f 7$ had LOD scores of 3.28 and 3.13 in the ZA/XDXBIL and ZA/XXD-BIL, with the DWR alleles increasing spikelet fertility by $11.3 \%$ and $6.9 \%$, respectively. The other common QTL $q R f 10.1$ had LOD scores of 3.70 and 6.30 in the ZA/XDX-BIL and ZA/XXD-BIL, with the O. rufipogon alleles enhancing spikelet fertility by $9.1 \%$ and $6.2 \%$, respectively. The remaining QTLs were $q R f 3, q R f 5.1$, and $q R f 10.2$, detected in the ZA/XDX-BIL, and $q R f 1.4$ and $q R f 5.2$ detected in the ZA/XXD-BIL. Individually, these QTLs contributed 4.9\%$44.3 \%$ of the phenotypic variance and had additive effects ranging from $4.0 \%$ to $17.2 \%$. The trait-enhancing alleles were again all derived from DWR.

\section{Discussion}

The exploitation of heterosis, such as by using $\mathrm{F}_{1}$ hybrids, is one of the most significant genetic achievements in agriculture. Nuclear $R f$ genes complement the male-sterile phenotype that arises with CMS, allowing the use of the CMS system for hybrid seed production. Molecular mapping of the $R f$ genes provides a powerful tool to develop restorer lines avoiding extensive testcrossing with CMS lines [41]. In this study, QTL analysis for fertility restoration of the DA-, DWR-, and ID-type CMS systems was conducted using two
O. sativa $\times$ O. rufipogon BIL populations. In total, 16 QTLs were detected, three for DWR-type CMS, six for DA-type CMS, and seven for ID-type CMS. It is notable that all 16 QTLs detected for fertility restoration of DA-, DWR-, and IDtype CMS had the enhancing alleles derived from DWR. The use of the favorable $O$. rufipogon QTL alleles would facilitate breeding of restorer lines in hybrid rice breeding.

Six of the QTLs detected in this study were located in genomic regions where QTLs for fertility restoration of the same type of CMS have been reported, including three of the six QTLs for fertility restoration of DA-type CMS and three of the seven QTLs for fertility restoration of ID-type CMS (Table 2). For DA-type CMS, $q R f 1.2$ colocated with a QTL for fertility restoration reported by Xie et al. [34]; qRf10.2 colocated with a QTL for spikelet fertility reported by Xie et al. [19, 34], which also corresponded to $R f 3$ and $R f 4$ for WA-type CMS [42-46], and $q R f 5.2$ overlapped with $q R f-5$ identified by Xie et al. [19]. For ID-type CMS, $q R f 1.4$ colocated with $q R f 5$ reported by Shen et al. [35]; qRf10.1 and $q R f 10.2$ located to both sides of $R f-4$ detected by Li et al. [14], which is also involved in restoring fertility of WA-type CMS. Recently, the gene Rf4, which restores WA-type CMS near the QTL $q R f 10.2$ (Figure 1) and encodes a pentatricopeptide repeatcontaining protein, has been cloned $[23,24]$. Thus, it may be considered that these QTLs are consistently detected across different environments and genetic backgrounds. In addition, $q R f 1.3, q R f 3, q R f 7, q R f 9$, and $q R f 5.1$ did not colocate with QTLs previously reported for fertility restoration of other CMS types and could be considered as new fertility restoration loci. These QTLs provide not only good candidates for QTL fine-mapping and cloning, but also novel genetic source for the breeding of restorer line in rice. 

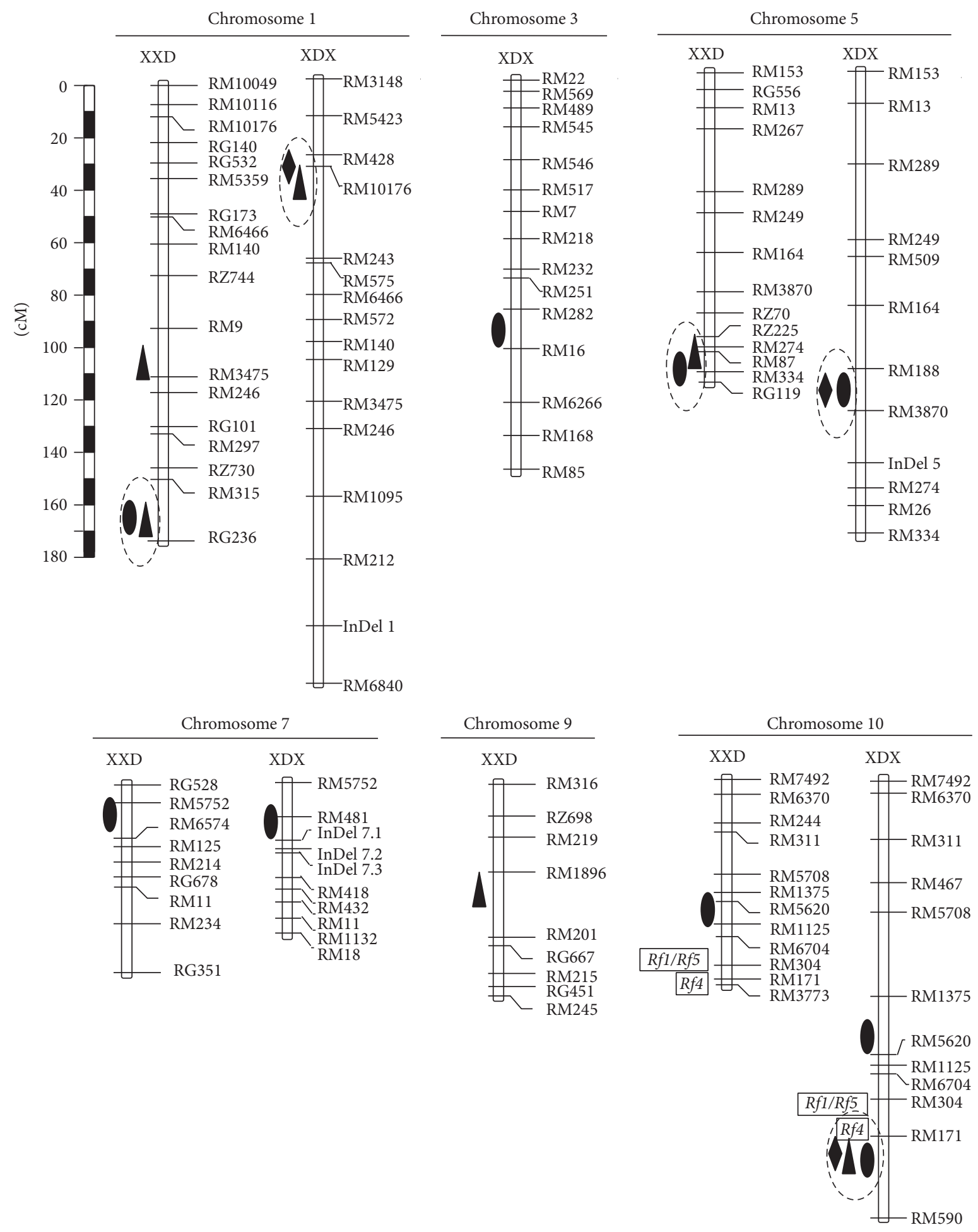

Zhong 9A

Xieqingzao A

DongB11A

FIGURE 1: Chromosomal positions of the QTLs conferring fertility restoration for DA-, DWR-, and ID-type CMS. XXD and XDX represent the backcross inbred lines of Xieqingzao (XB)//XB/Dongxiang wild rice (DWR) and XB//DWR/XB, respectively. 
Similar to the phenomena reported in many other studies, clustering of QTLs for fertility restoration was evident in the present study. Eleven of the QTLs detected in present study were located in five clusters distributed on three chromosomes (Figure 1). QTLs conferring fertility restoration for DA-, DWR-, and ID-type CMS were located in interval RM171-RM590 on chromosome 10. QTLs conferring fertility restoration for two CMS types were located in each of the other clusters, including two clusters each on chromosomes 1 and 5. These $R f$ loci clusters are of great importance for developing sustainable hybrid rice because they allow for the use of diverse CMS cytoplasmic sources and thus avoid the genetic vulnerability of monoculture in rice agriculture. QTL clusters $q R f 1.1$ for DA- and DWR-type CMS; $q R f 1.4$ and $q R f 5.2$ for DA- and ID-type CMS; and $q R f 10.2$ for three CMS types were clustered in four regions that have been previously reported (Table 2). Therefore, particular attention should be given to the QTL cluster $q R f 5.1$ in future studies of QTL finemapping and cloning.

The XXD BILs were previously conducted for mapping of QTLs for grain yield traits [37]. Of the 23 QTLs for yield components detected by Huang et al. [37], qTNSP9 was located in the region corresponding to $q R f 9$, and the allelic direction for fertility restoration was identical to the total number of spikelets per panicle. It was suggested that the $R f$ locus $q R f 9$ might contribute to grain yield in rice.

Interestingly, major $R f$ genes for other CMS systems in rice, such as $R f 1$ for BT-type CMS, $R f 4$ for WA-type CMS, $R f$ $D 1(t)$ for Dianl-type CMS, Rf5 for HL-type CMS, and $q R f$ 10-2 for DA-type CMS, were detected on the same region of chromosome 10 [17, 19, 25, 45-47] and were closely linked to form a gene cluster. Cloning of the $R f$ genes and sequence analysis reported by Tang et al. [24] and Hu et al. [25] revealed that the fertility restorer locus Rfla was identical to the locus $R f 5$ but different from the locus $R f 4$. In the present study, the major QTL $q R f 10.2$ for three CMS types was located in the same chromosomal region as $R f 4$. Further studies are required to define the allelic relationship between these genes.

For both DWR- and DA-type CMS, the genetic effect of $q R f 10.2$ was more pronounced than that of $q R f 1.1$. Similar results were reported for WA-type CMS in that the effect of $R f 4$ appeared to be larger than that of $R f 3$ and inheritance of both $R f$ genes resulted in compounded effects [18, 46, 48, 49]. The mitochondrial gene WA352 was recently described in wild rice and is associated with WA-, ID-, and DA-type CMS, suggesting that $R f 3(q R f 1.1)$ and $R f 4(q R f 10.2)$ are effective for restoring the fertility of DA-, ID-, and WA-type CMS [8]. This indicates that the two major $R f$ loci are associated with the different CMS/Rf systems in DA-, DWR-, and WAtype CMS. These results should lead to positive outcomes from alloplasmic line breeding and the introduction of DWR $R f$ genes into cultivated rice using advanced backcross QTL analysis in hybrid rice breeding programs.

When QTL results from the same mapping population were compared, the number and genetic effects of the identified QTLs varied amongst three CMS types, suggesting that different $R f$ alleles interact with each form of CMS in an independent manner. This study has provided novel information on the effects of O. rufipogon QTL alleles ( $q R f 1.1$, $q R$ f5.1, and $q R f 10.2)$ in DWR-type CMS. The results are in agreement with those reported by Li et al. [31], who claimed that $R f$ alleles coevolve with CMS. That is, wild rice lines that exhibit CMS may also carry the most useful genetic resources for associated fertility restoration. The O. rufipogon QTL alleles identified here provide good candidate $R f$ genes that can be used to develop new restorer lines for hybrid rice breeding and also further our understanding of the origin and evolution of $R f$ genes in rice.

\section{Competing Interests}

All authors have not declared any competing interests.

\section{Acknowledgments}

This research was supported by a grant from the National Natural Science Foundation of China (no. 31360327), the Key Projects of Jiangxi Education Department (no. KJLD12059), the Collaborative Innovation Programs of Modern Agricultural Scientific Research of Jiangxi Province (no. JXXTCX2015001002), and the Key Research and Development Programs of Jiangxi Province (no. 20161ACF60022).

\section{References}

[1] K. Miura, M. Ashikari, and M. Matsuoka, "The role of QTLs in the breeding of high-yielding rice," Trends in Plant Science, vol. 16, no. 6, pp. 319-326, 2011.

[2] Q. Qian, L. B. Guo, S. M. Smith, and J. Li, "Breeding high-yield superior quality hybrid super rice by rational design," National Science Review, vol. 3, no. 3, pp. 283-294, 2016.

[3] L.-P. Yuan, "Development of hybrid rice to ensure food security," Rice Science, vol. 21, no. 1, pp. 1-2, 2014.

[4] X. Huang, S. Yang, J. Gong et al., "Genomic analysis of hybrid rice varieties reveals numerous superior alleles that contribute to heterosis," Nature Communications, vol. 6, article 6258, 2015.

[5] G.-F. Fu, C.-X. Zhang, Y.-J. Yang et al., "Male parent plays more important role in heat tolerance in three-line hybrid rice," Rice Science, vol. 22, no. 3, pp. 116-122, 2015.

[6] X. Qin, Q. Huang, H. Xiao et al., "The rice DUF1620-containing and WD40-like repeat protein is required for the assembly of the restoration of fertility complex," New Phytologist, vol. 210, no. 3, pp. 934-945, 2016.

[7] T. Kazama, E. Itabashi, S. Fujii, T. Nakamura, and K. Toriyama, "Mitochondrial ORF79 levels determine pollen abortion in cytoplasmic male sterile rice," The Plant Journal, vol. 85, no. 6, pp. 707-716, 2016.

[8] D. P. Luo, H. Xu, Z. L. Liu et al., "A detrimental mitochondrialnuclear interaction causes cytoplasmic male sterility in rice," Nature Genetics, vol. 45, no. 5, pp. 573-577, 2013.

[9] J. Hu, W. Huang, Q. Huang et al., "Mitochondria and cytoplasmic male sterility in plants," Mitochondrion, vol. 19, pp. 282-288, 2014.

[10] J. M. Li and L. P. Yuan, "Hybrid rice: genetics, breeding, and seed production," Plant Breeding Reviews, vol. 17, pp. 15-64, 2000.

[11] J.-Z. Huang, Z.-G. E, H.-L. Zhang, and Q.-Y. Shu, "Workable male sterility systems for hybrid rice: genetics, biochemistry, molecular biology, and utilization," Rice, vol. 7, article 13, 2014. 
[12] S.-H. Cheng, J.-Y. Zhuang, Y.-Y. Fan, J.-H. Du, and L.-Y. Cao, "Progress in research and development on hybrid rice: a superdomesticate in China," Annals of Botany, vol. 100, no. 5, pp. 959966, 2007.

[13] S. Fujii and K. Toriyama, "Molecular mapping of the fertility restorer gene for $m s-C W$-type cytoplasmic male sterility of rice," Theoretical and Applied Genetics, vol. 111, no. 4, pp. 696701, 2005.

[14] L.-J. Li, H.-P. Zhou, X.-D. Zhan, S.-H. Cheng, and L.-Y. Cao, "Mapping of rice fertility-restoring genes for ID-type cytoplasmic male sterility in a restorer line R68," Rice Science, vol. 15, no. 2, pp. 157-160, 2008.

[15] P. B. Li, G. C. Su, F. C. Feng, P. Wang, S. Yu, and Y. He, "Mapping of minor quantitative trait loci (QTLs) conferring fertility restoration of wild abortive cytoplasmic male sterility and QTLs conferring stigma exsertion in rice," Plant Breeding, vol. 133, no. 6, pp. 722-727, 2014.

[16] W. C. Huang, J. Hu, C. C. Yu et al., "Two non-allelic nuclear genes restore fertility in a gametophytic pattern and enhance abiotic stress tolerance in the hybrid rice plant," Theoretical and Applied Genetics, vol. 124, no. 5, pp. 799-807, 2012.

[17] T. Komori, T. Yamamoto, N. Takemori, M. Kashihara, H. Matsushima, and N. Nitta, "Fine genetic mapping of the nuclear gene, $R f-1$, that restores the BT-type cytoplasmic male sterility in rice (Oryza sativa L.) by PCR-based markers," Euphytica, vol. 129, no. 2, pp. 241-247, 2003.

[18] M. Sattari, A. Kathiresan, G. B. Gregorio, and S. S. Virmani, "Comparative genetic analysis and molecular mapping of fertility restoration genes for WA, Dissi, and Gambiaca cytoplasmic male sterility systems in rice," Euphytica, vol. 160, no. 3, pp. 305$315,2008$.

[19] J.-K. Xie, J.-Y. Zhuang, Y.-Y. Fan, G.-Q. Tu, Y.-W. Xia, and K.-L. Zheng, "Mapping of fertility-restoring genes with main effects and epistatic effects for CMS-DA in rice," Acta Genetica Sinica, vol. 29, no. 7, pp. 616-621, 2002 (Chinese with English abstract).

[20] T. Komori, S. Ohta, N. Murai et al., "Map-based cloning of a fertility restorer gene, $R f-1$, in rice (Oryza sativa L.)," The Plant Journal, vol. 37, no. 3, pp. 315-325, 2004.

[21] Z. H. Wang, Y. J. Zou, X. Y. Li et al., "Cytoplasmic male sterility of rice with Boro II cytoplasm is caused by a cytotoxic peptide and is restored by two related PPR motif genes via distinct modes of mRNA silencing," The Plant Cell, vol. 18, no. 3, pp. 676-687, 2006.

[22] E. Itabashi, N. Iwata, S. Fujii, T. Kazama, and K. Toriyama, "The fertility restorer gene, $R f 2$, for Lead Rice-type cytoplasmic male sterility of rice encodes a mitochondrial glycine-rich protein," The Plant Journal, vol. 65, no. 3, pp. 359-367, 2011.

[23] T. Kazama and K. Toriyama, "A fertility restorer gene, Rf4, widely used for hybrid rice breeding encodes a pentatricopeptide repeat protein," Rice, vol. 7, no. 1, article 28, pp. 1-5, 2014.

[24] H. W. Tang, D. P. Luo, D. G. Zhou et al., "The rice restorer Rf4 for wild-abortive cytoplasmic male sterility encodes a mitochondrial-localized PPR protein that functions in reduction of WA352 transcripts," Molecular Plant, vol. 7, no. 9, pp. 1497-1500, 2014.

[25] J. Hu, K. Wang, W. C. Huang et al., "The rice pentatricopeptide repeat protein RF5 restores fertility in Hong-Lian cytoplasmic male-sterile lines via a complex with the glycine-rich protein GRP162," The Plant Cell, vol. 24, no. 1, pp. 109-122, 2012.

[26] W. C. Huang, C. C. Yu, J. Hu et al., "Pentatricopeptide-repeat family protein RF6 functions with hexokinase 6 to rescue rice cytoplasmic male sterility," Proceedings of the National Academy of Sciences of the United States of America, vol. 112, no. 48, pp. 14984-14989, 2015.

[27] S. Fujii and K. Toriyama, "Suppressed expression of retrograderegulated male sterility restores pollen fertility in cytoplasmic male sterile rice plants," Proceedings of the National Academy of Sciences of the United States of America, vol. 106, no. 23, pp. $9513-$ 9518, 2009.

[28] L. Bazrkar, A. J. Ali, N. A. Babaeian et al., "Tagging of four fertility restorer loci for wild abortive-cytoplasmic male sterility system in rice (Oryza sativa L.) using microsatellite markers," Euphytica, vol. 164, no. 3, pp. 669-677, 2008.

[29] X.-D. Luo, L.-F. Dai, J.-F. Cao et al., "Identification and molecular cytology analysis of cold tolerance introgression lines derived from Oryza sativa L. mating with O. rufipogon Griff," Euphytica, vol. 187, no. 3, pp. 461-469, 2012.

[30] F. T. Zhang, F. L. Cui, L. X. Zhang et al., "Development and identification of a introgression line with strong drought resistance at seedling stage derived from Oryza sativa L. mating with Oryza rufipogon Griff,' Euphytica, vol. 200, no. 1, pp. 1-7, 2014.

[31] S. Q. Li, G. H. Yang, S. B. Li, Y. S. Li, Z. Y. Chen, and Y. G. Zhu, "Distribution of fertility-restorer genes for wild-abortive and Honglian CMS lines of rice in the AA genome species of genus Oryza," Annals of Botany, vol. 96, no. 3, pp. 461-466, 2005.

[32] J. Chen, H. U. R. Bughio, D. Z. Chen et al., "Development of chromosomal segment substitution lines from a backcross recombinant inbred population of interspecific rice cross," Rice Science, vol. 13, no. 1, pp. 15-21, 2006.

[33] X. C. Xiao, Y. J. Wang, S. J. Xiao et al., "Comparison of heterosis in major agronomic characters between $\mathrm{F}_{1}$ hybrids of Dong B11A and those of IR58025A," Hybrid Rice, vol. 19, no. 2, pp. 1920, 2004 (Chinese with English abstract).

[34] J. K. Xie, J. Y. Zhuang, Y. Y. Fan et al., "Mapping of fertilityrestoring gene for Dwarf-wild-abortive CMS in rice," Chinese Journal of Rice Science, vol. 15, no. 3, pp. 161-164, 2001 (Chinese with English abstract).

[35] Y. W. Shen, Z. Q. Guan, J. Lu et al., "Linkage analysis of a fertility restoring mutant generated from CMS rice," Theoretical and Applied Genetics, vol. 97, no. 1-2, pp. 261-266, 1998.

[36] K. L. Zheng, N. Huang, and J. Bennet, "PCR-based markerassisted selection in rice breeding," IRRI Discussion Paper Series No. 12, IRRI, Manila, Philippines, 1995.

[37] D. R. Huang, J. Chen, L. J. Hou et al., "Identification of QTLs for yield traits in the $\mathrm{BC}_{1} \mathrm{~F}_{5}$ population of Xieqingzao B//Xieqingzao B/Dongxiang wild rice," Journal of Agricultural Biotechnology, vol. 16, no. 6, pp. 977-982, 2008 (Chinese with English abstract).

[38] E. S. Lander, P. Green, J. Abrahamson et al., "MAPMAKER: an interactive computer package for constructing primary genetic linkage maps of experimental and natural populations," Genomics, vol. 1, no. 2, pp. 174-181, 1987.

[39] S. C. Wang, C. J. Basten, and Z. B. Zeng, Windows QTL cartographer 2.5, Department of Statistics, North Carolina State University, Raleigh, NC, USA, 2012, http://statgen.ncsu.edu/qtlcart/ WQTLCart.htm.

[40] S. R. McCouch and CGSNL, "Gene nomenclature system for rice," Rice, vol. 1, no. 1, pp. 72-84, 2008.

[41] R. El-Namaky, S. Sedeek, Y. D. Moukoumbi, R. Ortiz, and B. Manneh, "Microsatellite-aided screening for fertility restoration genes $(R f)$ facilitates hybrid improvement," Rice Science, vol. 23, no. 3, pp. 160-164, 2016. 
[42] A. Ahmadikhah and G. I. Karlov, "Molecular mapping of the fertility-restoration gene $R f 4$ for WA-cytoplasmic male sterility in rice," Plant Breeding, vol. 125, no. 4, pp. 363-367, 2006.

[43] R. C. Jing, X. M. Li, P. Yi, and Y. Zhu, "Mapping fertilityrestoring genes of rice WA cytoplasmic male sterility using SSLP markers," Botanical Bulletin of Academia Sinica, vol. 42, no. 3, pp. 167-171, 2001.

[44] U. Ngangkham, S. K. Parida, S. De et al., "Genic markers for wild abortive (WA) cytoplasm based male sterility and its fertility restoration in rice," Molecular Breeding, vol. 26, no. 2, pp. 275292, 2010.

[45] P. B. Suresh, B. Srikanth, V. H. Kishore et al., "Fine mapping of $R f 3$ and $R f 4$ fertility restorer loci of WA-CMS of rice (Oryza sativa L.) and validation of the developed marker system for identification of restorer lines," Euphytica, vol. 187, no. 3, pp. 421435, 2012.

[46] F. Y. Yao, C. G. Xu, S. B. Yu et al., "Mapping and genetic analysis of two fertility restorer loci in the wild-abortive cytoplasmic male sterility system of rice (Oryza sativa L.)," Euphytica, vol. 98, no. 3, pp. 183-187, 1997.

[47] X. L. Tan, Y. L. Tan, Y. H. Zhao et al., "Identification of the $R f$ gene conferring fertility restoration of the CMS Dian-type 1 in rice by using simple sequence repeat markers and advanced inbred lines of restorer and maintainer," Plant Breeding, vol. 123, no. 4, pp. 338-341, 2004.

[48] J. Cai, Q. P. Liao, Z. J. Dai et al., "Allelic differentiations and effects of the $R f 3$ and $R f 4$ genes on fertility restoration in rice with wild abortive cytoplasmic male sterility," Biologia Plantarum, vol. 57, no. 2, pp. 274-280, 2013.

[49] J. Cai, Z. J. Dai, H. T. Zhu et al., "Comparative analysis of fertility restoration genes for WA, Y, and DA cytoplasmic male sterility in rice," Biologia Plantarum, vol. 58, no. 2, pp. 241-246, 2014. 

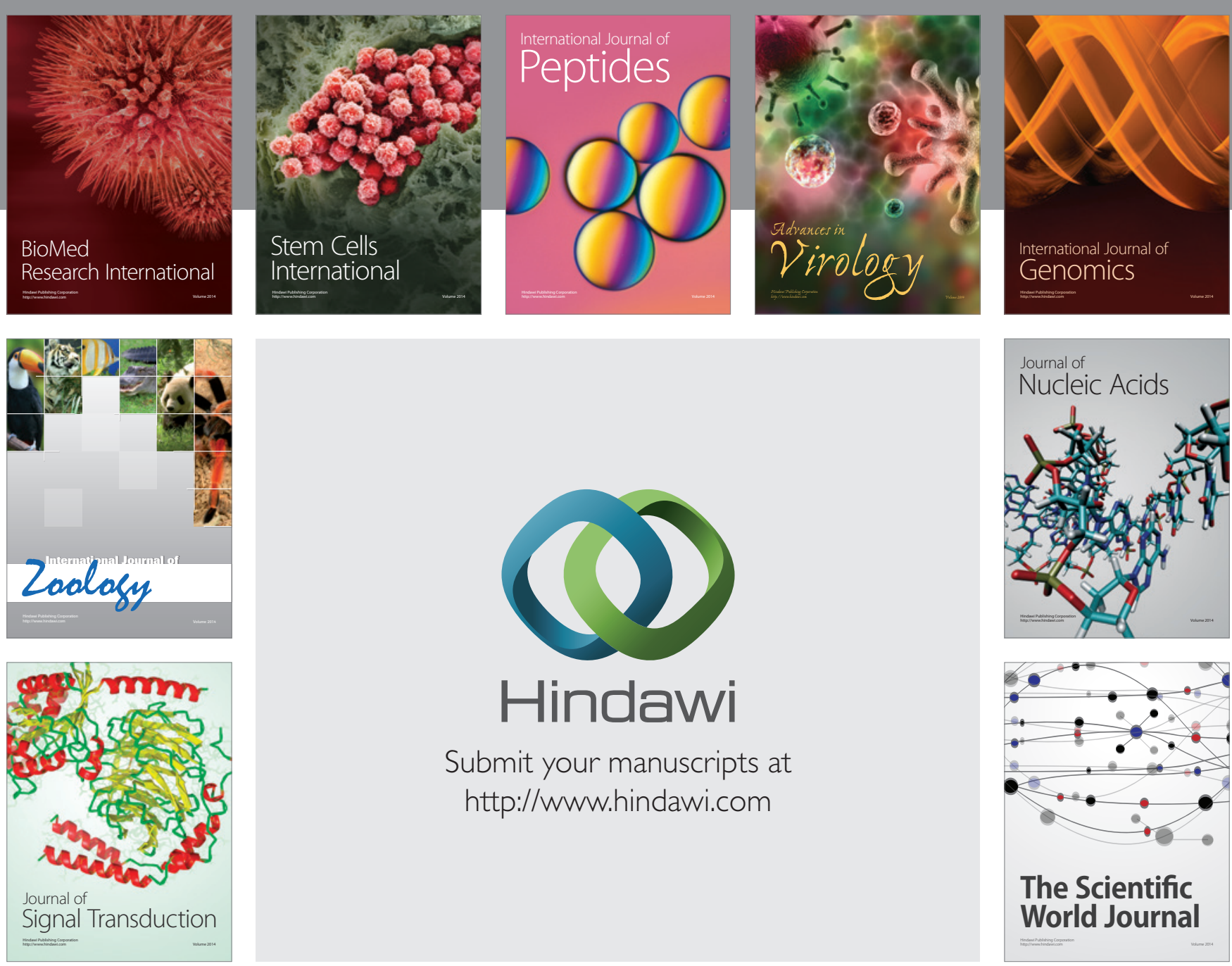

Submit your manuscripts at

http://www.hindawi.com
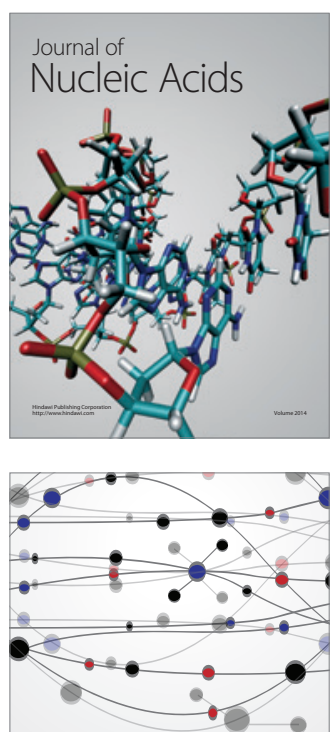

The Scientific World Journal
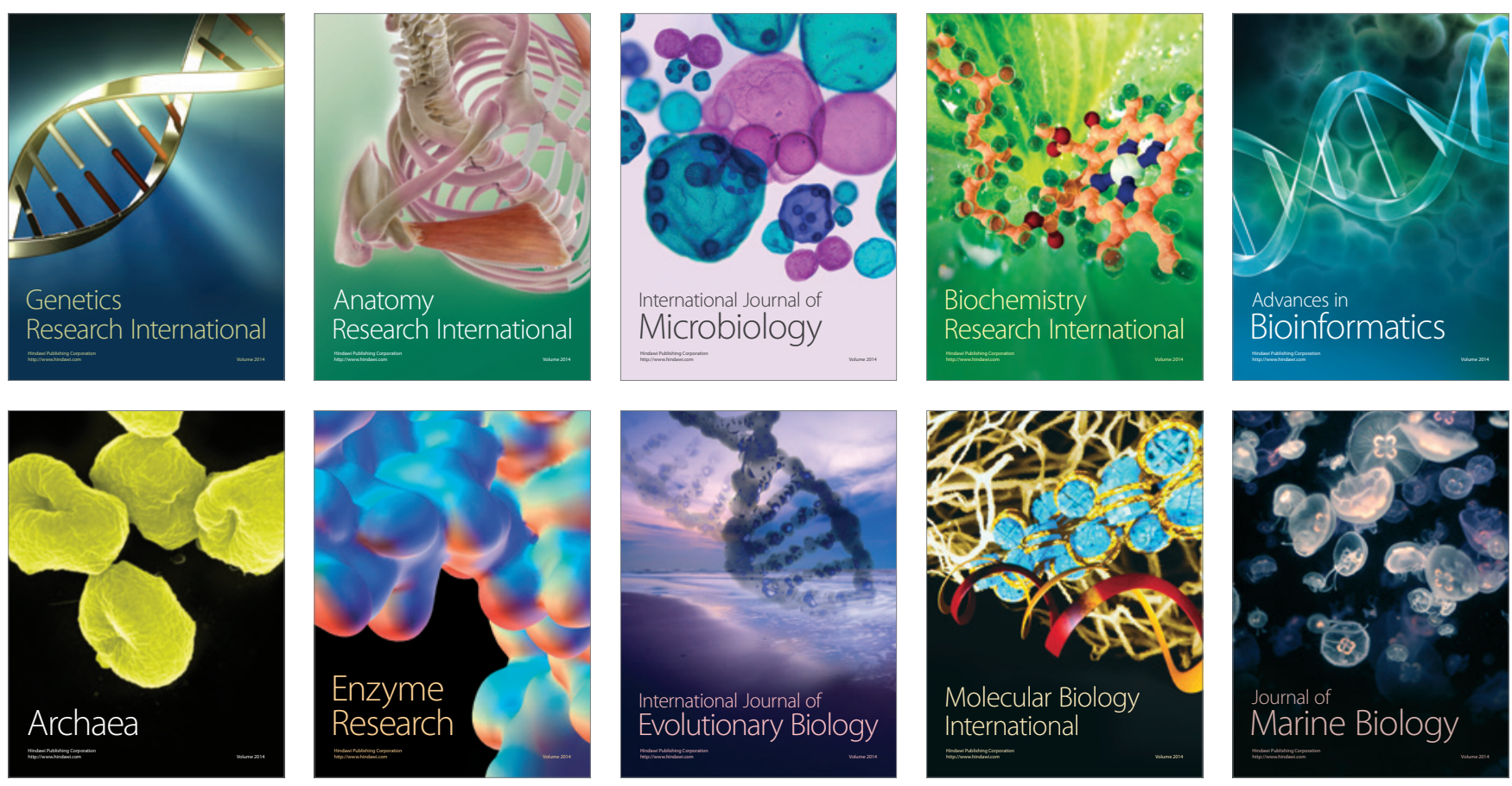